\title{
LE RICADUTE SULLA PROGRAMMAZIONE SANITARIA
}

\section{Falcitelli $\mathbf{N}$.}

\section{Centro Studi Fondazione Smith Kline, Roma}

Il sistema sanitario italiano presenta oggi una sanità in evoluzione dalla quale emerge una nuova attenzione per l'identificszione del bisogno sanitario della popolazione, attraverso l'individuazione di metodologie, di strumenti e di rinnovati assetti istituzionali, capaci di rispondere alle nuove richieste di domanda di salute del Paese.

I dati statistici e la loro aggregazione danno diverse chiavi di lettura, diversi spaccati di una società che ha ormai diffusamente recepita l'etica del benessere come parametro generale della tutela della salute, ma che è tuttora condizionata da aree di malessere del sistema sotto il profilo delle risorse, degli assetti istituzionali, della funzionalità e della individuazione dei campi e delle modalità di intervento.

La ricerca di strumenti e di metodologie particolari può ritenersi certamente espressione di una nuova sensibilità che ha come obiettivo quello della "produzione di salute" in luogo del tradizionale approccio produttivistico legato alle prestazioni assistenziali.

A tal fine, nell'ultimo periodo, è stata avviata una operazione certamente difficile, ma indispensabile, legata ai livelli essenziali di assistenza: l'identikit della mappa dei bisogni.

Per il momento, ma è già importante, si tratta di un avvio più di intenti che di risultati; d'altro canto è pur vero che non si possono sperare risultati se non c'è chiarezza e conoscenza reale della situazione e dei fenomeni sottostanti.

La ricerca dei diversi fattori va assumendo connotati sempre più precisi, passando gradualmente dalla fase della definizione dei principi fondamentali a quella della progettualità di strategie globali e di attività specifiche.

Basta qui ricordare l'indicazione dell'ultima legge finanziaria (quella per l'anno 2005) che prevede, tra l'altro, la fissazione di "standard qualitativi, strutturali, tecnologici, di processo e possibilmente di esiti, e quantitativi di cui ai livelli essenziali di assistenza". In quest'ottica si è mossa l'indagine conoscitiva delle strutture di diagnostica microbiologa distribuite sul territorio nazionale.come condizione preliminare per la realizzazione di un progetto programmatorio che, partendo dall'analisi della situazione esistente, fosse in grado di suggerire considerazioni e proposte capaci di affrontare le esigenze legate alla più moderna visione del sistema sanitario italiano.

Grande importanza assume, qui, il processo di pro- 
grammazione che caratterizza l'intero Servizio sanitario italiano. Adottato come metodo ordinario di governo e di sviluppo del servizio sanitario, la programmazione è certamente presente a livello di dibattito politico e culturale, ma è ancora non completamente presente a livello operativo, proprio perché carente del momento della conoscenza, ossia della individuazione corretta dei bisogni attraverso un adeguato sistema informativo che dai dati epidemiologici tragga coerenti indicazioni sulle strategie da adottare. 